\title{
Learning-Related Plasticity of Temporal Coding in Simultaneously Recorded Amygdala-Cortical Ensembles
}

\author{
Stephen E. Grossman, ${ }^{1,3}$ Alfredo Fontanini, ${ }^{1,2,3}$ Jeffrey S. Wieskopf, ${ }^{3}$ and Donald B. Katz ${ }^{1,2,3}$ \\ ${ }^{1}$ Volen National Center for Complex Systems, ${ }^{2}$ Department of Psychology, and ${ }^{3}$ Program in Neuroscience, Brandeis University, Waltham, Massachusetts \\ 02454
}

Emotional learning requires the coordinated action of neural populations in limbic and cortical networks. Here, we performed simultaneous extracellular recordings from gustatory cortical (GC) and basolateral amygdalar (BLA) neural ensembles as awake, behaving rats learned to dislike the taste of saccharin [via conditioned taste aversion (CTA)]. Learning-related changes in single-neuron sensory responses were observed in both regions, but the nature of the changes was region specific. In GC, most changes were restricted to relatively late aspects of the response (starting $\sim 1.0 \mathrm{~s}$ after stimulus administration), supporting our hypothesis that in this paradigm palatability-related information resides exclusively in later cortical responses. In contrast, and consistent with data suggesting the amygdala's primary role in judging stimulus palatability, CTA altered all components of BLA taste responses, including the earliest. Finally, learning caused dramatic increases in the functional connectivity (measured in terms of cross-correlation peak heights) between pairs of simultaneously recorded BLA and GC neurons, increases that were evident only during taste processing. Our simultaneous assays of the activity of single neurons in multiple relevant brain regions across learning suggest that the transmission of taste information through amygdala-cortical circuits plays a vital role in CTA memory formation.

Key words: taste; gustatory cortex; basolateral amygdala; conditioned taste aversion; single-unit; electrophysiology

\section{Introduction}

Perception and learning necessarily involve coordinated action among distributed groups of neurons, but such relationships have seldom been investigated in real time. Conditioned taste aversion (CTA), a simple learning paradigm whereby a formerly preferred taste becomes unpalatable after being paired with gastric distress (Nachman and Ashe, 1973; Bermudez-Rattoni et al., 1986; Gallo et al., 1992; Rollins et al., 2001), offers a unique window into systems-level properties of learning, because it vitally involves both basolateral amygdala (BLA) (Nachman and Ashe, 1974; Rollins et al., 2001; Reilly and Bornovalova, 2005; Wang et al., 2006) and gustatory cortex (GC) (Gallo et al., 1992; Schafe and Bernstein, 1998; Berman and Dudai, 2001; Stone et al., 2005), and because the speed with which taste aversions are learned makes it feasible to maintain isolations of single neurons from before until after learning using chronic microwire techniques (Katz et al., 2001b).

CTA training changes both GC (Yasoshima et al., 1995b; Yasoshima and Yamamoto, 1998) and BLA (Buresova et al., 1979; Yasoshima et al., 1995a) taste responses, but the properties of

Received Sept. 5, 2007; revised Feb. 4, 2008; accepted Feb. 5, 2008.

This work was supported by National Institute on Deafness and Other Communication Disorders Grants DC 008720 (S.E.G.), DC-006666 (D.B.K.), and DC-008885 (A.F.). A.F. was also supported by the Sloan-Swartz Center for Theoretical Neuroscience at Brandeis. We thank the members of the Katz Laboratory as well as Leslie Griffith for their valuable input.

This article is freely available online through the $J$ Neurosci Open Choice option.

Correspondence should be addressed to Donald B. Katz, Volen National Center for Complex Systems, MS 013, Brandeis University, 415 South Street, Waltham, MA 02454. E-mail: dbkatz@brandeis.edu.

D0I:10.1523/JNEUROSCI.4063-07.2008

Copyright $\odot 2008$ Society for Neuroscience $\quad$ 0270-6474/08/282864-10\$15.00/0 learning-related BLA and GC responses have received little study. It is reasonable to expect that learning-related changes in the two structures should differ. Palatability-related information only appears in GC between 0.8 and $1.0 \mathrm{~s}$ after taste administration (Katz et al., 2001a); CTA, which changes taste palatability without affecting actual taste quality (Berridge and Robinson, 1998), would therefore be predicted to change only these later aspects of GC taste responses (Fontanini and Katz, 2006). Indirect evidence, meanwhile, suggests that activity in BLA, which is known to code palatability (Nishijo et al., 1998), may drive CTA-related activity and plasticity in GC (Escobar et al., 1998a,b; Escobar and Bermudez-Rattoni, 2000; Miranda et al., 2002; Ferreira et al., 2005). Thus, learning should change earlier aspects of BLA responses than GC responses [i.e., learning should change BLA responses produced $<1 \mathrm{~s}$ after stimulus administration; for similar logic in fear conditioning, see Quirk et al. (1997), Armony et al. (1998), and Repa et al., 2001]. These data also suggest the novel hypothesis that CTA learning should be reflected in increased functional connectivity between BLA and GC (see also Bauer et al., 2007).

Here, we examine taste responses in simultaneously recorded ensembles of BLA and GC neurons held from before to after learning, and present evidence supporting this set of hypotheses. As expected, learning-related plasticity is restricted almost exclusively to late aspects of GC responses. Learning-related changes in BLA, meanwhile, demonstrate very short latencies; new BLA responses appear within $100 \mathrm{~ms}$ of taste delivery [before taste information has even reached GC (Di Lorenzo and Schwartzbaum, 1982; Katz et al., 2001a)]. Finally, the functional connectivity between BLA and GC neurons, assayed during taste processing, more than doubles with CTA learning.

These results reveal the relationship between single neurons 
simultaneously recorded from distinct parts of the distributed CTA network, and as such they offer important clues to the systems-level properties of perception and learning.

\section{Materials and Methods}

Subjects

Male and female Long-Evans rats ( $n=18 ; 250-300 \mathrm{~g}$ at time of surgery) served as subjects in this study. Animals were maintained on a $12 \mathrm{~h}$ light/dark schedule and were given ad libitum access to chow and water, unless otherwise specified. All methods complied with the Brandeis University Institutional Animal Care and Use Committee guidelines.

\section{Surgery}

Rats were anesthetized using an intraperitoneal injection of a ketamine/ xylazine/acepromazine mixture $(100 \mathrm{mg} / \mathrm{kg}, 5.2 \mathrm{mg} / \mathrm{kg}$, and $1 \mathrm{mg} / \mathrm{kg}$, respectively), with supplemental intraperitoneal injections administered as needed. Each anesthetized rat was placed in a standard stereotaxic device, where its scalp was excised, and holes were bored in its skull for the insertion of 0-80 ground screws and electrode bundles. Multielectrode bundles [ 16 nichrome microwires attached to a microdrive (Katz et al., 2001b)] were inserted $0.5 \mathrm{~mm}$ above GC [anteroposterior (AP), 1.4 $\mathrm{mm}$; mediolateral (ML) $-5 \mathrm{~mm}$; dorsoventral (DV), $-4.5 \mathrm{~mm}$ from dura] and BLA (AP, $-3 \mathrm{~mm}$; ML, $-5.1 \mathrm{~mm}$; DV, $-6.5 \mathrm{~mm}$ from dura). Once in place, the assemblies were cemented to the skull, along with two intraoral cannulas (IOCs) (Fontanini and Katz, 2006), using dental acrylic. Rats were given $7 \mathrm{~d}$ to recover from the surgery.

\section{CTA protocol}

Adaptation. After recovery and throughout the CTA protocol, rats were maintained on $45 \mathrm{~min} / \mathrm{d}$ water restriction to ensure adequate motivation to drink. Each rat was adapted to the conditioning context for $9 \mathrm{~d}$ : for the first 2 of these days, it was placed in the testing chamber for $30 \mathrm{~min}$; for each of the next $7 \mathrm{~d}$, the fluid delivery tubing was affixed to its head. Rats were adapted both to slow infusions of $5 \mathrm{ml}$ of water (delivered through one IOC at a rate of $0.5 \mathrm{ml} / \mathrm{min}$ ) and to $50 \mu \mathrm{l}$ pulses ( 35 per session) delivered at a frequency of $3 / \mathrm{min}$.

Training. After the adaptation sessions, each rat received a single training session. This session was identical to adaptation sessions, except that water was replaced with $0.15 \%$ saccharin as an oral stimulus. Video of the rat's facial responses to saccharin delivery was recorded to digital video disc. Five minutes after the termination of saccharin exposure, the rat was given an intraperitoneal injection of $\mathrm{LiCl}(0.15 \mathrm{M}, 2 \%$ body weight $)$ to induce gastric distress. Rats were returned to their home cages immediately after $\mathrm{LiCl}$ injection, to ensure that they would not form conditioned place aversions to the testing chamber.

Testing. Twenty-four hours after training, the rat received a testing session, identical to the training session, in which the acquisition of CTA was measured and neural responses to saccharin were again recorded.

\section{Control conditions}

An additional group of rats $(n=4)$ received "sham" CTAs, in which a benign $0.9 \%$ saline solution was substituted for $\mathrm{LiCl}$ in posttraining injections. Neural data collected from these rats allowed us to test whether learning-independent aspects of the protocol (repeated sessions of taste delivery, i.p. injections, etc) caused modifications of taste responses in and of themselves. Another group of control rats $(n=2)$ received CTAs to $0.1 \mathrm{M} \mathrm{NaCl}$ (delivered orally) before receiving sham conditioning with saccharin; neural data collected from these rats allowed us to test the possibility that the experience of sickness might change taste responses. A third group of rats $(n=3)$ received CTAs to saccharin, but were then exposed to $\mathrm{NaCl}$ as a taste on the testing day (to avoid confusion, we will refer to $\mathrm{NaCl}$ used as an oral/taste stimulus as " $\mathrm{NaCl}$," and refer to sham i.p. injections as "saline"). These rats received a second testing session with saccharin $24 \mathrm{~h}$ later. This condition allowed us to test whether successful CTAs were solely related to the taste delivered during the testing session, and not to contextual elements of the experimental procedures (no neural activity was recorded in this last control group).

In all regards other than those described above, experimental procedures used with control rats were identical to those used with CTA rats.

\section{CTA quantification}

Rats produce stereotyped, easily observed behaviors when an aversive stimulus is in the mouth [i.e., gapes (Grill and Norgren, 1978; Spector et al., 1988)]. A significant increase in the number of gapes elicited by saccharin after training (by paired $t$ test) served as evidence for learning here, as it has in many previous studies (Breslin et al., 1992).

\section{Electrophysiology}

Neural signals were collected from BLA and GC during taste sampling in both training and testing sessions. Differential recordings were fed into a parallel processor capable of digitizing up to 32 signals at $40 \mathrm{kHz}$ simultaneously (Plexon, Dallas, TX). Discriminable action potentials of no less than 3:1 S/N ratio were isolated on-line from each signal using an amplitude criterion in cooperation with a template algorithm. Discriminations were checked continuously throughout each session. Time-stamped records of stimulus onset and neuronal spikes were saved digitally, as were all sampled spike waveforms and the discrimination file (Nicolelis et al., 1997). Off-line reanalysis incorporating three-dimensional cluster cutting techniques confirmed and corrected on-line discriminations.

\section{Determining stable single-unit recordings across training/testing} days of CTA

To characterize the direct effects of CTA on individual neuronal taste responses, we examined neurons that remained stably isolated across the $24 \mathrm{~h}$ between prelearning and postlearning sessions. The stability of microwire bundles makes this feat feasible (Katz et al., 2001b). To statistically evaluate the stability of our unit isolations, average action potential waveforms from training and testing sessions were first mapped into a three-dimensional principal component space using commercially available spike-sorting software (Wavetracker; Plexon). Multivariate ANOVAs (mANOVAs) allowed a comparison of the waveforms recorded on a particular electrode during training and testing days of CTA. As a control comparison, training sessions were divided into thirds, and mANOVAs were used to compare the first and last third of the spikes recorded within the single session. These sessions were at most $12.5 \mathrm{~min}$ long; thus, the compared sets of waveforms in this control condition were typically separated by a 4 min interval, an interval that all but ensures waveform stability. Waveforms were only described as coming from the same single neuron across training and testing sessions if the multivariate $F$ value comparing the two sessions was in the same range as those for the withinsession comparisons (supplemental Fig. S1, available at www.jneurosci. org as supplemental material); in practice, this criterion led us to define neurons as having been held across learning only if the training and testing waveforms were determined to have a $<0.05$ probability of being different.

An empirical demonstration of the validity of this approach comes from recordings made from control rats: in the absence of learning, neurons deemed to have been held across sessions almost always responded the same to saccharin in tests separated by $24 \mathrm{~h}$ (see below).

\section{Detection of lick-rate modulation in somatosensory neurons}

Some neurons in GC respond to somatosensory stimulation of the tongue and oral cavity (Yamamoto et al., 1988). These neurons fire action potentials synchronized to the rhythmic $(6-9 \mathrm{~Hz})$ licking behavior produced by the rat during taste sampling (Katz et al., 2001a). This spectral signature allowed us to identify and then remove such neurons from further analysis, and thus to ensure that motor behaviors (which change with CTA) would not confound response plasticity related to taste quality and/or palatability coding. Power spectral density plots (256 frequency values sampled between 2 and $30 \mathrm{~Hz}$ ) were used to determine which neurons, across an entire session, produced peaks between 6 and 9 $\mathrm{Hz}$. Peaks in this range of at least 1.5 SDs above the average $2-30 \mathrm{~Hz}$ power were considered somatosensory neurons, and these neurons were removed from analysis.

\section{Analysis of $G C$ and BLA taste responses}

Time-averaged responses (firing rate averaged across the first $2.5 \mathrm{~s}$ after taste delivery) were compared with the averaged baseline firing rate (1.5 $\mathrm{s}$ of prestimulus activity) using paired $t$ tests, with taste responsiveness determined by a significant change from baseline (note that although 
learning sometimes caused subtle changes in spontaneous activity, all results described here were qualitatively similar for raw and normalized responses). $\alpha$ values were set to 0.01 , unless spontaneous and evoked firing rates were very low (i.e., $<1$ spike/s), in which case a slightly less conservative $\alpha$ value of 0.05 was used. This adjustment was made because fewer spikes result in a smaller sample from which to run inferential statistics. To determine the temporal properties of a taste response, we ran similar analyses on responses divided into consecutive $250 \mathrm{~ms}$ bins. Finally, we performed a dynamic moving window analysis on the peristimulus time histograms (PSTHs) of each neuron (Katz et al., 2001a), to determine the onset and offset times at which firing rates changed from baseline (and the durations of these modulations).

Initial analyses of how learning affected saccharin responses were performed in full neural ensembles, without regard to whether or not the neurons were held across sessions, using the $250 \mathrm{~ms}$ bin analysis described above. The percentage of training session neurons that produced significant saccharin responses in each bin was compared with the percentage of testing session neurons that produced significant saccharin responses.

\section{Analysis of neurons held from before until after learning}

To more rigorously and directly examine the effects of CTA on GC and BLA taste responses, we analyzed the subset of units recorded across both days of CTA training/testing. Paired $t$ tests were used to compare firing rate responses on consecutive days, first using $2.5 \mathrm{~s}$ and then $250 \mathrm{~ms}$ bins. Statistical $p$ values were set as described above.

Principal components analysis (PCA) was performed to compare the time courses of pre- and post-CTA population responses to saccharin (Stopfer et al., 03). For each neuron, the response to the stimulus was averaged across trials and divided in consecutive $100 \mathrm{~ms}$ bins. PCA was performed on two $n \times m$ matrices (one for prelearning and the other for postlearning), where $n=$ the number of neurons and $m=$ the number of time bins ( 25 bins of $100 \mathrm{~ms}$ duration, for a total poststimulus time of $2.5 \mathrm{~s})$. The time course of the similarity was computed by measuring the Euclidean distance between homologous points of the two matrices in multidimensional space.

\section{Analysis of functional connectivity between BLA and GC}

Functional connectivity was determined by measuring the crosscorrelations between spike trains of simultaneously recorded BLA and GC neuron pairs (held throughout real or "sham" learning). Pairs were constructed of each pair of held neurons; if, for instance, two BLA and three GC neurons were held across learning in one rat, six amygdalacortical neuron pairs were analyzed for that rat. Correlated activity was examined for both spontaneous activity and saccharin responses in CTA rats, and for saccharin responses in control rats. To ensure a stable analysis of cross-correlations during saccharin responses, $4 \mathrm{~s}$ of poststimulus time was used (Katz et al., 2002). The absolute value of the crosscorrelation peak was measured within -500 and $+500 \mathrm{~ms}$ of the spike of each "master" neuron of the pair. To remove cross-correlations that could be attributed to common drive onto both neurons (i.e., that did not have to do with actual interactions between the recorded neuron pair), the "optimal shift predictors" (cross-correlations made after the trials of one of the neurons are shuffled; essentially cross-correlations between the two PSTHs with trial-to-trial coherence ignored) were subtracted from the cross-correlations made from the original data (Perkel et al., 1967).

\section{Histology}

After the experimental sessions, subjects were deeply anesthetized and perfused through the heart with saline followed by $10 \%$ formalin in saline. Seven seconds of DC current $(7 \mu \mathrm{A})$ were passed through selected microwires in preparation for staining. Brains were removed and immersed in a sucrose formalin mixture, where they remained, refrigerated, until fixed. Sections $(40 \mu \mathrm{m})$ cut through the implanted areas on a freezing microtome were stained with Prussian blue for ferrous deposits blasted off of the electrode tips, and counterstained with cresyl violet for cell bodies.

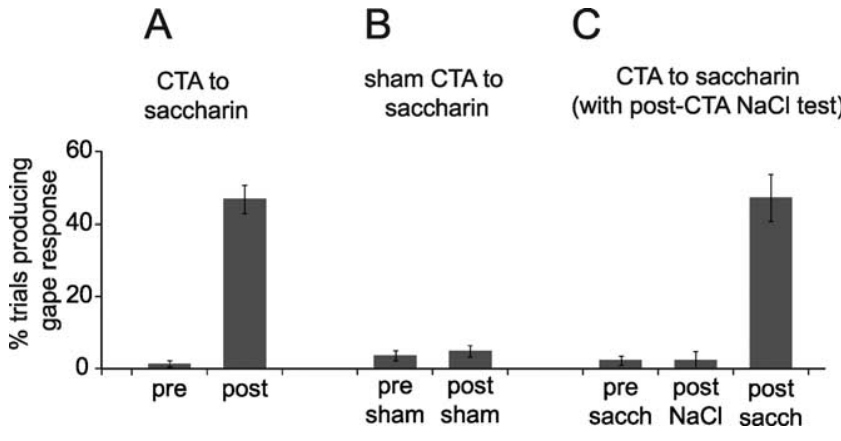

Figure 1. Associative learning. $A$, Naive rats ("pre-") seldom gape in response to saccharin delivery ( $y$-axis shows the percentage of deliveries that caused gapes), but trained rats ("post-") do. B, Sham training, in which the nonemetic saline replaces the emetic LiCl, does not cause increased likelihood of gapes. C, CTAs to saccharin do not generalize to other palatable stimuli (in this case, $\mathrm{NaCl}$ ), but even after testing with $\mathrm{NaCl}$, saccharin continues to cause gapes.

\section{Results}

\section{Associative taste learning}

After adaptation to the testing/recording chamber, rats received a training session of saccharin infusions through an IOC, followed immediately by intraperitoneal injections of either an emetic ( $\mathrm{LiCl})$ or neutral (saline) solution. We evaluated the development of CTA by comparing the number of gapes [orofacial behaviors that communicate an animal's disliking of a taste (Berridge, 2000)] produced in response to taste administration before training to those produced after training (Spector et al., 1988). Figure $1 \mathrm{~A}$ shows that CTA rats (i.e., rats that received saccharin/ $\mathrm{LiCl}$ pairings) seldom gaped in response to the sweet taste of saccharin during training sessions, but that they frequently gaped during subsequent testing sessions. The difference between prelearning and postlearning responses was significant $\left(t_{(8)}=11.4\right.$; $p<0.0001)$. Gapes did not emerge in sham-trained rats (i.e., rats that received saccharin/saline pairings) (Fig. $1 B$ ). The difference between these groups was highly significant $\left(t_{(13)}=8.8\right.$; $p<$ $0.0001)$.

Saccharin-LiCl pairings cause few gapes in response to orally infused $\mathrm{NaCl}(t<1)$, but these same animals, tested $1 \mathrm{~d}$ later, showed the expected aversion to saccharin $\left(t_{(2)}=7.3 ; p<0.02\right)$ (Fig. 1C). Meanwhile, animals conditioned with $\mathrm{NaCl}-\mathrm{LiCl}$ pairings did gape in response to oral $\mathrm{NaCl}$ during testing sessions (data not shown). This proves that our training paradigm induced a true associative CTA; that is, rats gained an aversion to the taste stimulus, and not to the testing situation.

\section{The multisite neural sample}

Spectral analysis (see Materials and Methods) revealed a subset of GC neurons with obvious somatosensory receptive fields. With these neurons removed (to limit the possibility that somatosensory responses accompanying learning-related oral behaviors might be confused with genuine learning-related responses), our total GC dataset was 123 neurons (76 recorded from 9 CTA rats, 47 from 6 control rats). Because basic analyses revealed no differences between the saccharin responses of control rats that received just sham conditioning and those of rats that received sham conditioning after induction of CTAs to $\mathrm{NaCl}$, data from these two control groups were combined.

Of the 76 GC neurons isolated from CTA rats, 51 (5.7 \pm 3.9 neurons/rat) were recorded during training sessions and 47 $(5.2 \pm 4.5)$ during testing sessions. Of these, $22(2.4 \pm 3.1)$ satisfied our criteria for being held across both sessions. Of the $47 \mathrm{GC}$ neurons from control rats, $40(6.7 \pm 3.8)$ were recorded during 
A

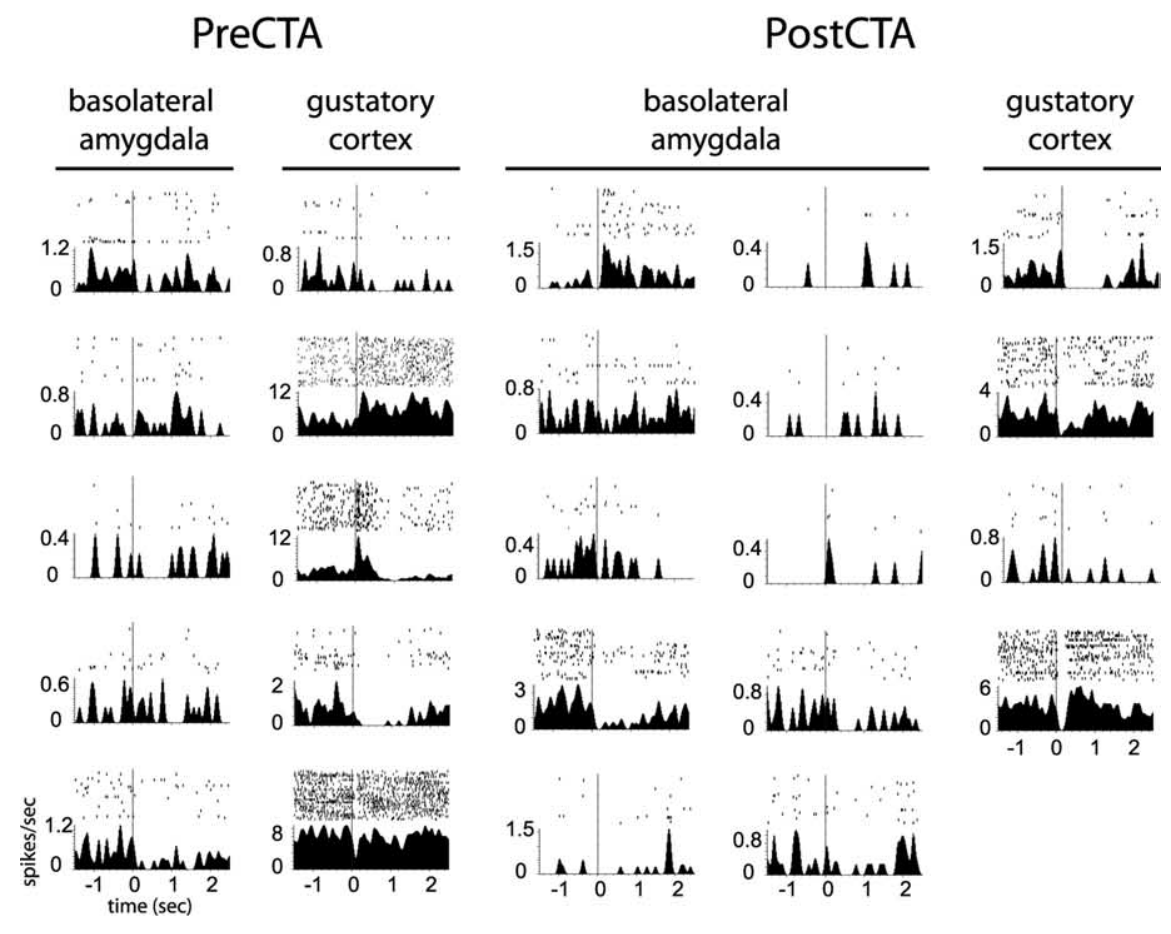

B gustatory cortex

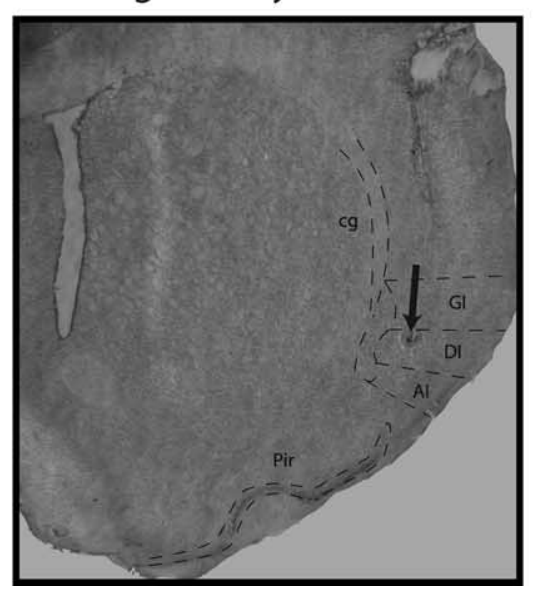

basolateral amygdala

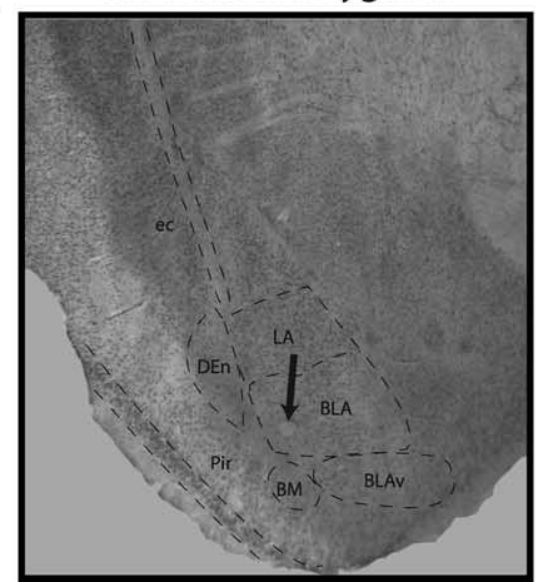

Figure 2. Neural data. A, A representative pair of ensembles containing simultaneously recorded gustatory cortical and basolateral amygdalar neurons, recorded before and after a CTA was established to saccharin. For each neuron, the PSTH shows the average firing rate ( $y$-axis) from -1.5 to $2.5 \mathrm{~s}$ after saccharin delivery (delivered via intraoral cannula at $t=0$; vertical dashed lines). Above each PSTH, raster plots display individual action potentials as single black dots. Rasters are divided into 35 rows beginning with trial 1 on top and extending downward chronologically. B, Sample histology, showing placement of electrode tips (lesion holes are marked with arrows). Left, Electrode placement within the granular and dysgranular insular cortex. Right, Electrode placement within the basolateral amygdala. Al, Agranular insular cortex; BLAv, ventral basolateral amygdala; BM, basomedial amygdala; $\mathrm{cg}$, cingulum; DEn, dorsal endopiriform cortex; DI, dysgranular insular cortex; ec, external capsule; $\mathrm{Gl}$, granular insular cortex; LA, lateral amygdala; Pir, piriform cortex.

training sessions and $41(6.8 \pm 4.1)$ during testing sessions; 34 of these neurons were held across both days of CTA.

A total of 77 BLA neurons were isolated (50 from 8 CTA rats, 27 from 6 control rats). Of the 50 neurons recorded from CTA rats, $34(4.3 \pm 4.9)$ were isolated during training sessions and 36 (4.5 \pm 4.9$)$ during CTA testing; 20 units $(2.5 \pm 3.7)$ were held across both days of CTA. Of the 27 control neurons, 17 (2.8 \pm 1.8) were recorded during training sessions and $20(3.3 \pm 1.9)$ during testing sessions; 10 of these neurons were held across both days of CTA. Figure 2 shows the saccharin responses of representative neural ensembles, one recorded during training (left) and the other during testing (right) sessions, from a single CTA rat, along with photomicrographs showing the locations of electrode tips from a representative rat.

\section{Taste responses in GC}

Responses to saccharin in training sessions were qualitatively similar to those observed previously in our lab: neuron responses typically changed across one or more of three distinct epochs of activity, reflecting somatosensory (taste presence), chemosensory (taste identity), and palatability information sequentially (Katz et al., 2001a; Fontanini and Katz, 2006).

When responses were averaged across 2.5 s of poststimulus time, $43 \%$ of the GC neural sample recorded during training sessions produced a saccharin response. Of neurons recorded during testing sessions, only $13 \%$ produced responses that were tonic enough to reach significance across $2.5 \mathrm{~s}$. Figure $3 A$, which shows the overall percentage of GC neurons that responded to saccharin in consecutive 250 $\mathrm{ms}$ bins separately for training and testing ensembles, reveals this drop to be the result of the fact that relatively few GC neurons recorded in testing sessions responded to saccharin in late (i.e., palatability-related) portions of the taste responses; this drop between the percentage of saccharin responses in the first and second seconds was significant $\left(t_{(8)}=\right.$ $3.82 ; p<0.01)$. In training sessions, meanwhile, the likelihood of saccharin responses was similar across bins $(\sim 30 \% /$ bin, $\left.t_{(8)}<1.0\right)$. The difference between the prelearning and postlearning distribution of saccharin responses was significant $\left(\chi_{(9)}^{2}=18.86 ; p=0.02\right)$, suggesting that learning, on average, may have truncated responses such that postlearning modulations were of insufficient length to be detected in $2.5 \mathrm{~s}$ averages. A moving-window analysis of firing-rate modulations supported this suspicion, showing that the average duration of GC saccharin responses dropped significantly, from $768 \pm 92 \mathrm{~ms}$ before learning to $427 \pm 56 \mathrm{~ms}$ afterward $\left(t_{(149)}=3.32 ; p<0.01\right)$

In control animals trained with pairings of saccharin and intraperitoneal saline (a nonemetic stimulus), training/testing differences in saccharin responses were minimal. Whereas true CTA training massively reduced the percentage of neurons that responded to saccharin, sham training did not (28 and $29 \%$ of GC neurons responded before and after training, respectively, in overall responses). Furthermore, similar percentages of GC neu- 
A

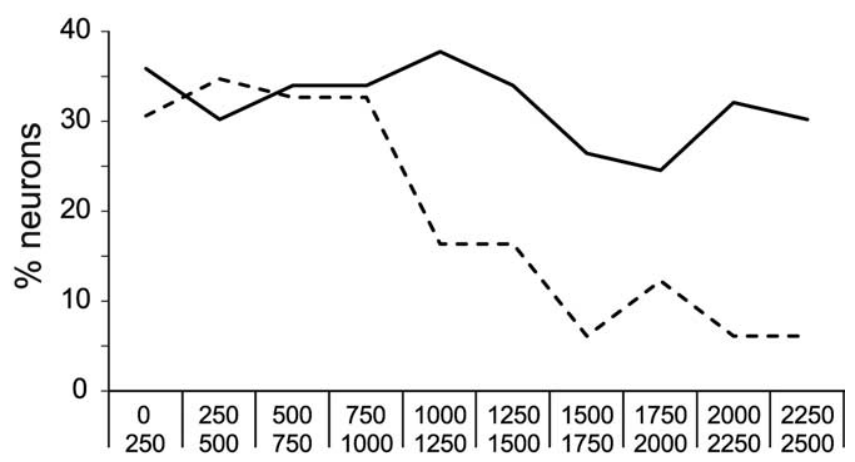

B

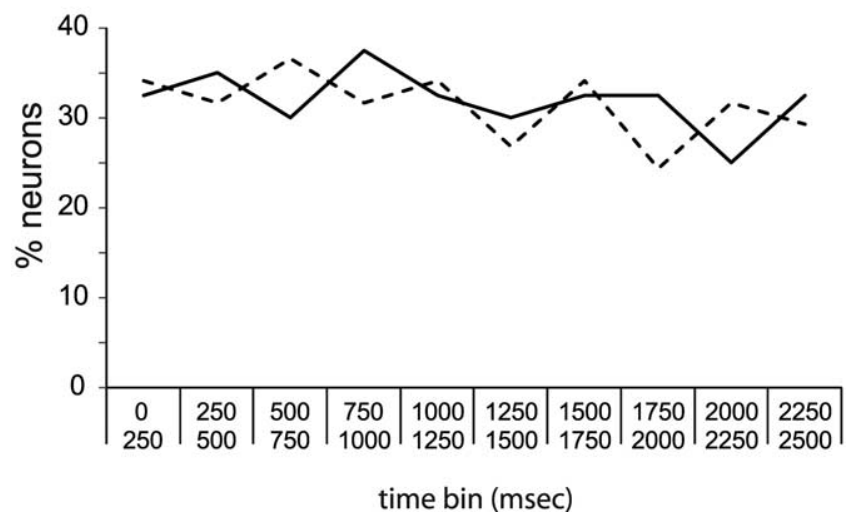

Figure 3. The likelihood of $\mathrm{GC}$ saccharin responses in training and testing sessions, examined independently. $\boldsymbol{A}$, The $x$-axis shows time (in $250 \mathrm{~ms}$ bins), and the $y$-axis shows the percentage of $G($ neurons showing significant responses to saccharin in ensembles recorded during training sessions (solid line) and ensembles recorded during testing sessions (dashed line). Similar percentages of neurons responded at all time points in the training ensembles, but in testing session ensembles there was a striking decrease in the percentage of saccharin responses starting at $\sim 1$ s. $\boldsymbol{B}$, Similarly divided data for neurons collected from control rats, showing that the changes in number of saccharin responses shown in $\boldsymbol{A}$ are a specific function of taste learning.

rons responded to saccharin in each $250 \mathrm{~ms}$ time period before and after sham conditioning. These results are shown in Figure $3 B$.

\section{CTA-induced GC response plasticity, examined directly in neurons held across training}

The above analysis tells us only that taste learning changes the percentage of neurons activated by saccharin, revealing nothing about its impact on specific responses themselves. A rigorous, direct analysis of the impact of CTA on GC responses can only be performed on the neurons held across (i.e., from before until after) learning. Such analysis showed that $\sim 32 \%$ of these $22 \mathrm{GC}$ neurons responded differently to saccharin after learning (Fig. $4 B$ ), and that this response plasticity consisted almost exclusively of changes emerging after $0.75 \mathrm{~s}$ of response time (Fig. $4 C, D$ ). This percentage of GC neurons changed by CTA is similar to the percentage of cortical and amygdalar neurons changed by fear conditioning (Quirk et al., 1995, 1997), and to the percentage of cerebellar cortical neurons showing learning-related responses during eyeblink conditioning (Katz and Steinmetz, 1997).

Only 3\% $(n=1)$ of the GC neurons held across sham conditioning in control rats changed across the training and testing sessions (Fig. $4 B$ ). In addition to demonstrating that response plasticity is purely a function of learning, this percentage, which is essentially identical to chance, drives home the validity of our unit isolation techniques; in the absence of learning (Fig. 1), the saccharin responses of held neurons are stable across $24 \mathrm{~h}$.

Figure $4 \mathrm{~A}$ shows the prelearning and postlearning PSTHs (light and dark gray, respectively) of three plastic neurons from CTA rats, with time periods of significant pre-post differences marked (horizontal lines). Each of the first two neurons produced significant responses to saccharin during the first $1.0 \mathrm{~s}$ after stimulus delivery (one excitatory, one inhibitory) that were largely unchanged by CTA. After this time point, however, their postlearning responses differed markedly from their prelearning responses. The learning-related plasticity shown by neuron 3 , meanwhile, began as early as $250 \mathrm{~ms}$ after taste stimulation, but even this change continued into later aspects of the response (Katz et al., 2001a; Fontanini and Katz, 2006). Figure 4C shows, for each held GC neuron that was changed by CTA, the time periods of significant pre-post differences (the neurons from Fig. $4 A$ are marked), and Figure $4 D$ summarizes these changes, demonstrating that learning-related response changes began appearing in the period leading up to $1 \mathrm{~s}$ after stimulus onset, and lasted through the period previously described as reflecting palatability coding.

Figure 4 suggests, but does not prove, that CTA specifically changes late aspects of GC population temporal codes (although the distribution appears far from uniform, the sample size is too small for significance). A PCA time-vector characterization (Stopfer et al., 2003) of saccharin responses in the GC neurons held across learning (supplemental Fig. S2 A, available at www. jneurosci.org as supplemental material) was therefore used to expose the overall effect of CTA on the population coding of saccharin, by showing how ensemble responses move through multidimensional space across time. Figure 5 summarizes the effect of learning on population coding, showing the difference between prelearning and postlearning responses at successive time points in the full multidimensional response space. Although present from the beginning of the responses, the difference between prelearning and postlearning saccharin coding increased significantly at the $1 \mathrm{~s}$ mark of the response $\left(t_{(24)}=3.87\right.$; $p<0.001)$. Thus, and as predicted by previous work in our laboratory (Fontanini and Katz, 2006), a learning-induced change in saccharin palatability (Bures et al., 1998) appears to be uniquely reflected in late epochs of GC taste activity leaving the chemosensory epoch (Katz et al., 2001a) relatively unchanged.

\section{Taste responses in BLA}

Figure $6 A$ shows, separately for neurons recorded in training and testing sessions, the percentage of BLA neurons that produced saccharin responses. As was true for GC (Fig. 3), the responses of prelearning BLA ensembles are spread relatively evenly across 250 ms bins ( $\sim 7 \% /$ bin $)$; in postlearning ensembles, moderately more saccharin responses were observed early (in the first $1.25 \mathrm{~s}$ ) than later, but this difference did not approach significance $(t<$ $1)$; nor did the difference between the distributions of responses before and after learning $\left(\chi^{2} p>0.7\right)$. Similar results were obtained for BLA saccharin responses from control rats (Fig. 6B): there were no significant differences between the percentage of neurons that responded to saccharin before and after sham training. In the small sample of sham neurons, there was an insignificant trend toward more early responses; this trend was likely just the result of random sampling. These data suggest one of two possibilities: either CTA had no impact on BLA saccharin responses or response plasticity was distributed such that there was 
A
GC neuron \#1

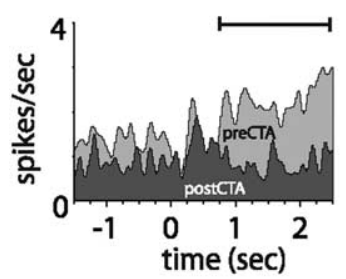

B

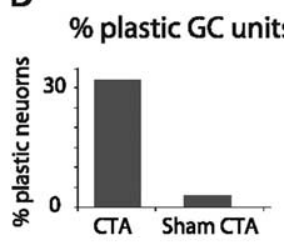

\begin{abstract}
C
\end{abstract}

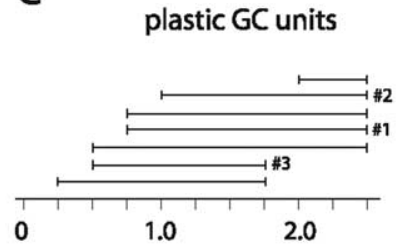

GC neuron \#2

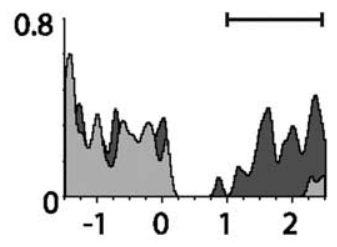

GC neuron \#3

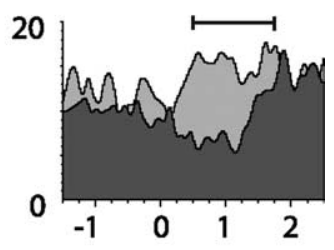

changed as a result of CTA, whereas not one single BLA neuron (out of 10) changed in control rats (Fig. $7 B$ ).

Learning-related changes in BLA responses tended to have short latencies, and to last for at least 1 full second (and beyond). Figure $7 A$ shows learning-related plasticity in three representative BLA neurons that could be directly observed undergoing learning-related plasticity; as in Figure $4 A$, light gray PSTHs are from prelearning sessions, dark gray PSTHs are from postlearning sessions, and horizontal lines show time bins of significant response plasticity. Learning-related changes had a very early onset and long duration for each neuron. Figure 7, $C$ and $D$, summarizes this analysis, showing that the majority of learning-related BLA changes were of very short onset [in some cases, starting earlier than the onset of any taste-related activity in GC in this paradigm (Katz et al., 2001a)] and long lasting. Other BLA changes caused by CTA were diverse in timing.

When the data from the BLA neurons held across learning were subjected to PCA, it was clear that prelearning and postlearning population codes for saccha-

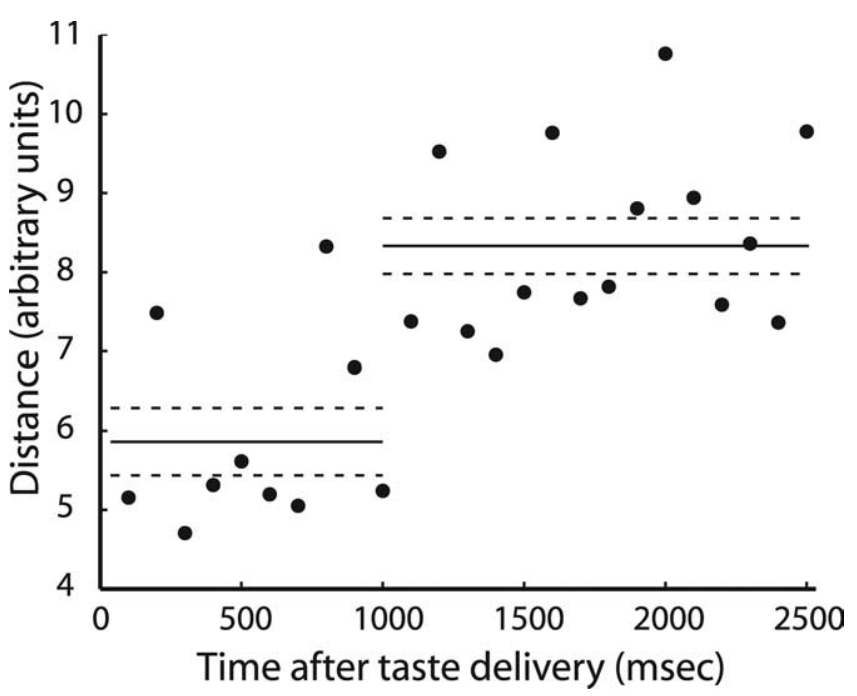

Figure 5. CTA-related changes in GC population coding of taste for neurons held across learning. The $y$-axis shows the Euclidean distance between matching time points in the PCA analysis computed on the unsmoothed, unreduced data for successive $100 \mathrm{~ms}$ bins ( $x$-axis); that is, these data show the difference between the prelearning and postlearning GC population codes for saccharin, at every point in stimulus-processing time. The solid horizontal lines represent the average distances computed for first poststimulus second and later time bins, respectively; the dashed lines are SE.

no overall change in the distribution of responses similar numbers of responses gained and lost at each time point). Further analysis demonstrated that the latter conclusion was correct.

\section{CTA-induced BLA response plasticity}

Again, a deeper and more direct understanding of the changes wrought by CTA resulted from an analysis of the BLA neurons held across (i.e., from before until after) learning. This analysis revealed that CTA learning does cause significant changes in BLA saccharin responses. In fact, $30 \%$ of the BLA neurons held across learning
A

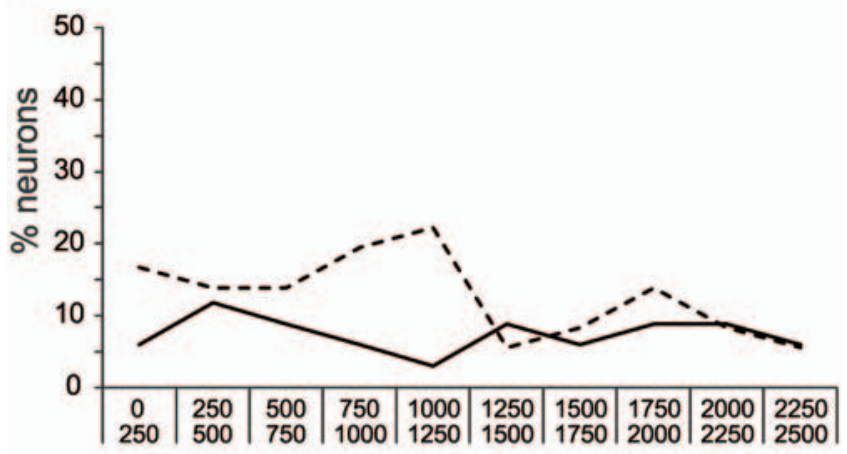

B

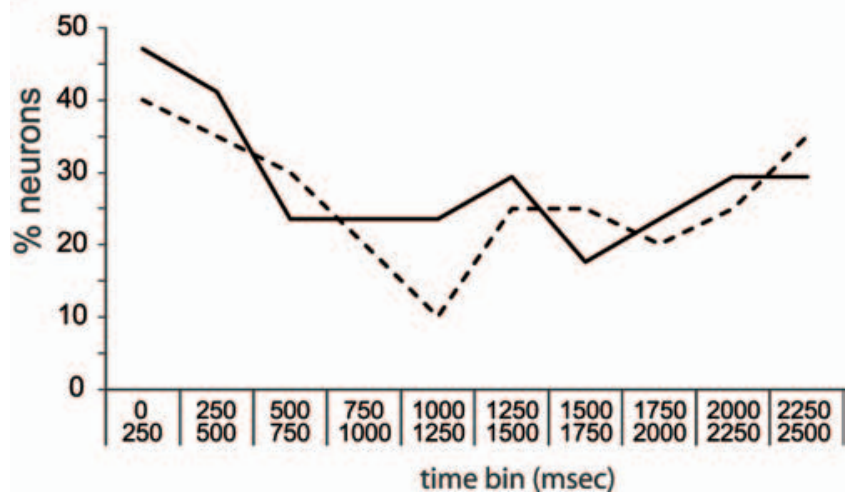

Figure 6. The likelihood of BLA saccharin responses in training and testing sessions, examined separately. $\boldsymbol{A}$, The $x$-axis shows time (in $250 \mathrm{~ms}$ bins), and the $y$-axis shows the percentage of BLA neurons showing significant responses to saccharin in ensembles recorded during training sessions (solid line) and ensembles recorded during testing sessions (dashed line) in CTA rats. No significant differences in the number of BLA neurons that responded to saccharin were visible. $\boldsymbol{B}$, Similar analysis for control rats. 
BLA neuron \#3

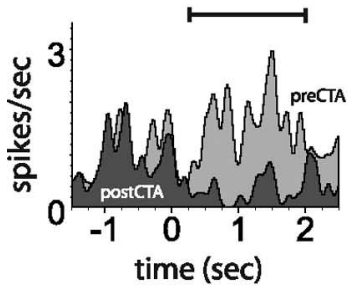

B $\%$ plastic BLA units

c
BLA neuron \#1

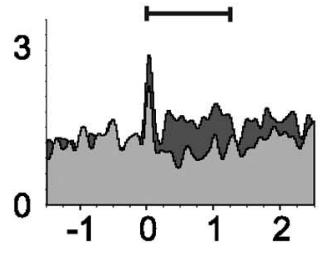

\section{plastic BLA units}

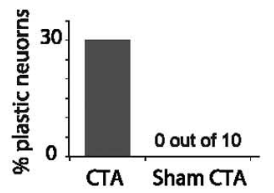

BLA neuron \#4

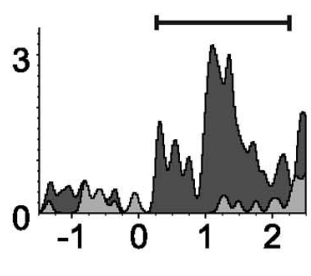

D

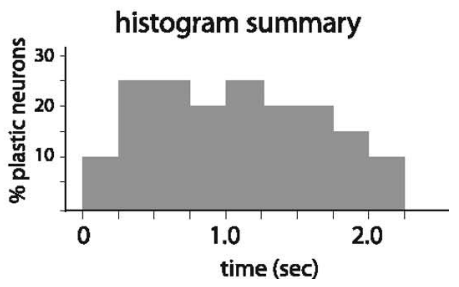

Figure 7. CTA-related plasticity in single BLA neurons held across learning. A, PSTHs of three representative BLA units held through training and testing sessions. Each example contains overlain saccharin PSTHs from before (light gray) and after (dark gray) learning. Solid horizontal lines above the PSTHs delineate periods of significant learning-related changes. $\boldsymbol{B}$, The overall percentage of $B L A$ neurons for which saccharin responses changed, for rats that received CTAs and for control rats. $C$, The time bins during which CTA changed saccharin responses (i.e., as for the solid horizontal lines in $\boldsymbol{A}$ ), for each plastic BLA neuron. The $x$-axis shows poststimulus time in $250 \mathrm{~ms}$ bins. The results for the neurons shown in $A$ are marked. $\boldsymbol{D}$, A summary of these data, showing the total percentage of held BLA units with modified firing at each time point.

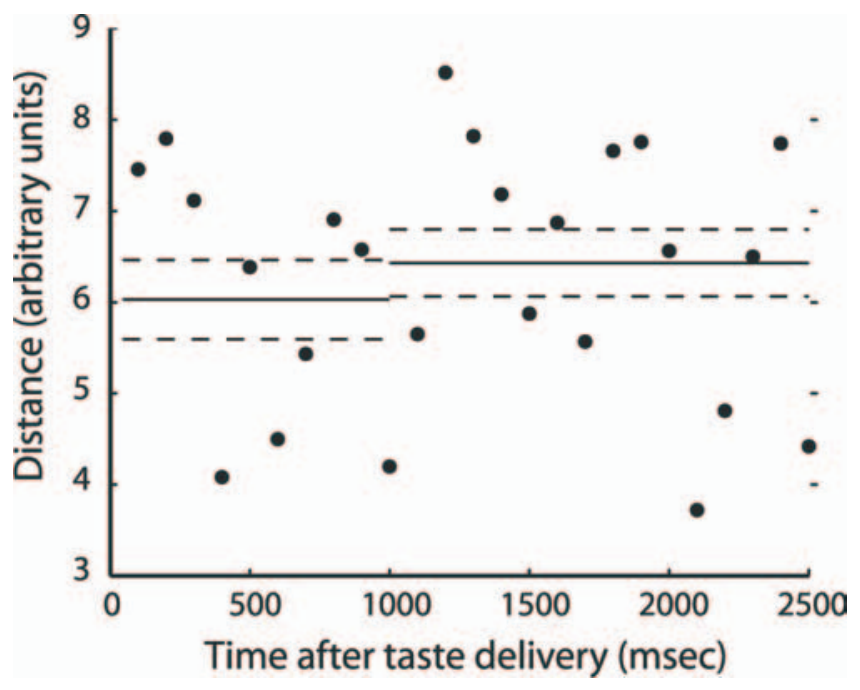

Figure 8. CTA-related changes in BLA population coding of taste for neurons held across learning. Euclidean distances between matching time points in the PCA analysis, computed on the unsmoothed, unreduced data. The $y$-axis shows the Euclidean distance between matching time points in the PCA analysis computed on the unsmoothed, unreduced data for successive 100 ms bins ( $x$-axis); that is, these data show the difference between the prelearning and postlearning BLA population codes for saccharin, at every point in stimulus-processing time. Conventions are as for Figure 5

rin were distinct and unrelated (supplemental Fig. S2 B, available at www.jneurosci.org as supplemental material). The responses were on separate trajectories from the earliest spikes and remained on these different trajectories throughout poststimulus time. Figure 8 demonstrates, in fact, that the distance between the prelearning and postlearning trajectories was high at the earliest time points, and that the trajectories did not appear to move with any particular relationship to each other across time. Saccharin coding in BLA was massively changed by CTA, which appears to have induced a wholesale reorganization of amygdalar processing.

\section{The relationship between BLA and GC during CTA learning}

The above analyses reveal that new, learning-related saccharin responses in BLA appear early in poststimulus time (Figs. 6, 7), whereas such new responses in GC have a later poststimulus onset (Figs. 3, 4). This accords well with studies suggesting that CTA may be underlain by plasticity in amygdala-cortical synapses (Escobar and Bermudez-Rattoni, 2000; Ferreira et al., 2005). For such a hypothesis to be considered feasible, however, it must be shown that CTA genuinely increases the functional connectivity between GC and BLA. We therefore analyzed the crosscorrelations between simultaneously recorded pairs of GC and BLA neurons during saccharin deliveries.

To ensure maximum interpretability, the analysis of cross-correlations between BLA-GC neuron pairs was restricted to those neurons held across both training and testing sessions. A total of 44 GC-BLA neuron pairs were used (each neuron could participate in multiple pairs; see Materials and Methods). Figure $9 A$ shows the prelearning and postlearning crosscorrelations for two representative amygdala-cortical pairs of neurons: each correlation function hovered close to 0 before training (green curves), and each deviated much further from 0 (i.e., showed enhanced functional connectivity) after training (red curves). To summarize this analysis, we calculated the difference between the postlearning and prelearning peak heights for each neuron pair (Fig. 9B, left bar). The sizes of crosscorrelation enhancements ranged from -0.07 to +0.44 (out of a maximum difference of +2 ), with fully $75 \%$ of the crosscorrelations becoming more positive with learning.

We performed the same analysis on spontaneous activity in CTA rats, and also on sham-trained rats. For neither of these control conditions did training increase functional connectivity (Fig. 9B, center and right bars): only $47 \%$ of the spontaneous or sham cross-correlations increased with learning (ranges: -0.07 to +0.02 , spontaneous; -0.09 to +0.06 , sham). A one-factor ANOVA for the data in Figure $9 B$ was highly significant $\left(F_{(2,100)}\right.$ $=3.09 ; p<0.01)$; the increase in functional connectivity observed during taste processing in the CTA rats was significantly different from that observed in either control condition (both $p<0.05, t$ tests). CTA increased amygdala-cortical functional connectivity, but this increase was specific to periods of taste coding.

Figure 10 shows the raw cross-correlation peak heights for the full sample of BLA-GC pairs, calculated during saccharin consumption (left) and during periods of spontaneous activity (right), before (white bars) and after (gray bars) learning. CTA more than doubled the average height of BLA-GC crosscorrelation peaks, while having no impact on spontaneous crosscorrelations. The prelearning versus postlearning comparison was significant $\left(t_{(42)}=2.1 ; p<0.05\right)$, and the postlearning, tasteprocessing cross-correlations were significantly higher than postlearning spontaneous cross-correlations $\left(t_{(42)}=2.1 ; p<\right.$ 
0.04). The difference between spontaneous prelearning and postlearning crosscorrelations was not significant $(p>$ 0.08).

In summary, CTA more than doubled the strength of functional connectivity between BLA and GC, but only in the context of taste processing itself. This, coupled with the temporal relationship between BLA and GC learning-related response changes, leads us to suggest that increased amygdalocortical communication is an important aspect of CTA learning.

\section{Discussion}

Here, we have shown that CTA changes the way that taste information flows through simultaneously recorded GC and BLA ensembles. Almost all of the learningrelated GC plasticity is localized to the time period previously related to palatability processing (Katz et al., 2001a; Fontanini and Katz, 2006). Examination of the subgroup of neurons held across learning confirms that pre- and post-CTA PSTHs are highly similar for the first $0.8-1.0 \mathrm{~s}$ and divergent afterward. Concurrently recorded BLA plasticity, meanwhile, has an extremely early onset and long duration. A doubling of amygdala-cortical functional connectivity is observed during post-CTA taste processing, a fact that, in conjunction with our other results, implies that taste learning increases information flow between BLA and GC.

Sham conditioning (substitution of a neutral i.p. injection for the emetic $\mathrm{LiCl}$ ) had no impact on either structure; furthermore, saccharin responses after induction of a CTA to a salty solution resembled those observed in naive or sham-conditioned rats. These controls make it clear that response plasticity was the result of CTA learning, and not of (1) exposure to the taste of saccharin; (2) loss of, or change of response in, well isolated neurons through time; (3) peritraining intraperitoneal injections; or (4) exposure to $\mathrm{LiCl}$. The observed response plasticity is, in fact, a specific function of taste learning.

The data support our contention that GC performs a feat known as multiplexing, processing first somatosensory, then chemosensory, and finally palatability-related aspects of the stimulus, in the time between taste administration and the appearance of palatability-specific behavioral responses (Travers and Norgren, 1986). Our previous work provided evidence of temporally specific information in GC codes (Katz et al., 2001a), and demonstrated that palatability-related attentional shifts preferentially change epochs of the GC responses that code palatability (Fontanini and Katz, 2006). Here, we show that CTA-related response plasticity in GC is restricted almost exclusively to this same epoch. Because CTA specifically changes the perceived palatability of saccharin, while leaving the taste quality itself unchanged (Frank et al., 2003), these results represent direct evidence that the late period of the cortical taste response is indeed reporting the processing of palatability.

We cannot, as of now, offer an explanation for the fact that most GC changes consisted of response reductions. It is clear that the area of insular cortex from which these recordings were made is vital for CTA learning (Stone et al., 2005), which suggests (although does not prove) that late saccharin responses did not merely "move elsewhere" in the brain; the most likely explana-
B

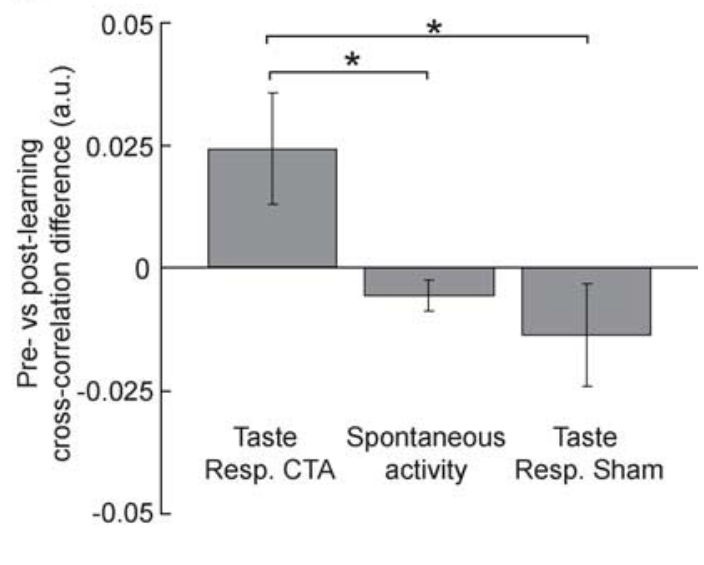


tor responses, which are easily identified as those that show whole-session spike trains modulated at 6-9 $\mathrm{Hz}$ lick rate, and which were removed from the dataset. Finally, the observed plasticity is appropriately timed to precede, not be preceded by, tasterelated behaviors in this preparation (Travers and Norgren, 1986). CTA-related plasticity almost assuredly represents changes in palatability processing in multiplexed GC temporal codes.

It is worth noting that rats can learn to avoid a taste with as little as $150 \mathrm{~ms}$ of exposure when drawing that taste from a lick spout (Halpern and Tapper, 1971), well before the emergence of palatability-related processing in our experiments. This fact likely reflects a difference between behavioral paradigms. When taste is delivered through a lick spout, the rat must make an approach behavior to acquire that taste. In our paradigm, meanwhile, tastes are delivered through the IOC; our CTA is a classical, as opposed to operant, conditioning paradigm. This difference is an important one, because approach behaviors activate a broad set of neural systems in anticipation of taste delivery (Yamamoto et al., 1988; Gutierrez et al., 2006). Anticipation changes basic aspects of taste activity (Nitschke et al., 2006), greatly decreasing the latency of taste responses in GC (Stapleton et al., 2006) and changing the circuits involved in CTA (Schafe et al., 1998; Wang et al., 2006). In an operant conditioning paradigm, we would expect palatability processing and learning-related response plasticity to both have shorter poststimulus latencies.

The amygdala, in contrast to GC, appears to be specialized for processing stimuli with intense hedonic valence (Nishijo et al., 1998; Small et al., 2003). As BLA is a direct recipient of taste information from the brainstem (Norgren and Leonard 1973) and the main output of hedonic information, it is reasonable to expect that CTA-induced neural changes should appear in the earliest portions of the BLA responses, much earlier than those observed in GC. This was confirmed in our data: in BLA, CTA modified firing rates as early as $0-100 \mathrm{~ms}$ after taste delivery, even earlier than the earliest taste responses detected in GC in our preparation (Katz et al., 2001a). These new BLA responses, which tended to be long lasting, are well timed to provide information about acquired palatability changes to GC.

Earlier studies indirectly suggest that information transfer from BLA to GC is a vital part of CTA learning. BLA output courses to several ingestion- and reward-related regions, including GC (Yamamoto et al., 1984; Ferreira et al., 2005). Furthermore, when tetanic stimulation is applied to BLA [a procedure that induces NMDA-dependent long-term potentiation in GC (Jones et al., 1999; Escobar et al., 1998a,b, 2002)] immediately before CTA induction, retention of the learning is enhanced (Escobar and Bermudez-Rattoni, 2000). A weak CTA can be enhanced by intra-BLA infusion of glutamate (Miranda et al., 2002), but this effect is blocked by NMDA receptor blockade in GC (Ferreira et al., 2005), again suggesting that amygdalocortical connections are an important part of CTA learning. Relatedly, a recent report (Bauer et al., 2007) shows that $\gamma$-oscillatory (35-45 $\mathrm{Hz}$ ) field potential coherence between BLA and rhinal cortex increases across $6 \mathrm{~d}$ of learning to respond to a visual cue paired with a food reward.

Our single-unit recordings from BLA and GC neurons provide direct evidence that shifts in hedonic values may be underlain by increased functional connectivity in the amygdalocortical pathway, and suggests that perceptual learning is specifically reflected in increased functional connectivity between brain regions. Cross-correlations between pairs of BLA and GC neurons increased (more than doubling) during posttraining presenta- tions of saccharin. Although this change is nonintuitive, given the reduction in response in GC caused by learning, it is easily incorporated into the overall characterization of CTA-induced plasticity: changes in functional connectivity are dissociable from changes in firing rate; if all of the "random" spikes are removed from two spike trains, for instance, the firing rates of both will go down but the cross-correlation between the two will increase.

The learning-related increase in functional connectivity was specific to taste processing (it was not observed during periods of spontaneous firing), and thus indicates a specific role for BLA-GC interactions during aversive saccharin processing. Together, these results suggest that amygdalocortical information transfer may be a vital part of conditioned taste aversions. How GC takes excitatory input from BLA and transforms it into inhibitions of activity is a question that will probably need to be answered by slice or in vivo intracellular experiments examining learning-induced changes in cortical microcircuitry.

These results of course investigate only a part of the circuitry involved in generating an experience-dependent taste percept. The importance and novelty of this work is that it directly shows that it is reasonable to characterize perception and perceptual learning in terms of "information flow" across distributed networks. Future work will continue to expand our understanding of the real-time multiarea interactions by recording from even larger networks.

\section{References}

Armony JL, Quirk GJ, LeDoux JE (1998) Differential effects of amygdala lesions on early and late plastic components of auditory cortex spike trains during fear conditioning. J Neurosci 18:2592-2601.

Bauer EP, Paz R, Pare D (2007) Gamma oscillations coordinate amygdalorhinal interactions during learning. J Neurosci 27:9369-9379.

Berman DE, Dudai Y (2001) Memory extinction, learning anew, and learning the new: dissociations in the molecular machinery of learning in cortex. Science 291:2417-2419.

Bermudez-Rattoni F, Grijalva CV, Kiefer SW, Garcia J (1986) Flavor-illness aversions: the role of the amygdala in the acquisition of taste-potentiated odor aversions. Physiol Behav 38:503-508.

Berridge KC (2000) Measuring hedonic impact in animals and infants: microstructure of affective taste reactivity patterns. Neurosci Biobehav Rev 24:173-198.

Berridge KC, Robinson TE (1998) What is the role of dopamine in reward: hedonic impact, reward learning, or incentive salience? Brain Res Brain Res Rev 28:309-369.

Breslin PA, Spector AC, Grill HJ (1992) A quantitative comparison of taste reactivity behaviors to sucrose before and after lithium chloride pairings: a unidimensional account of palatability. Behav Neurosci 106:820-836.

Bures J, Bermúdez-Rattoni F, Yamamoto T (1998) Conditioned taste aversion: memory of a special kind. Oxford: Oxford UP.

Buresova O, Aleksanyan ZA, Bures J (1979) Electrophysiological analysis of retrieval of conditioned taste aversion in rats. Unit activity changes in critical brain regions. Physiologia Bohemoslovaca 28:525-536.

Di Lorenzo PM, Schwartzbaum JS (1982) Coding of gustatory information in the pontine parabrachial nuclei of the rabbit: temporal patterns of neural response. Brain Res 251:245-257.

Escobar ML, Bermudez-Rattoni F (2000) Long-term potentiation in the insular cortex enhances conditioned taste aversion retention. Brain Res 852:208-212.

Escobar ML, Chao V, Bermudez-Rattoni F (1998a) In vivo long-term potentiation in the insular cortex: NMDA receptor dependence. Brain Res 779:314-319.

Escobar ML, Alcocer I, Chao V (1998b) The NMDA receptor antagonist CPP impairs conditioned taste aversion and insular cortex long-term potentiation in vivo. Brain Res 812:246-251.

Escobar ML, Alcocer I, Bermudez-Rattoni F (2002) In vivo effects of intracortical administration of NMDA and metabotropic glutamate receptors antagonists on neocortical long-term potentiation and conditioned taste aversion. Behav Brain Res 129:101-106.

Ferreira G, Miranda MI, De la Cruz V, Rodriguez-Ortiz CJ, Bermudez- 
Rattoni F (2005) Basolateral amygdala glutamatergic activation enhances taste aversion through NMDA receptor activation in the insular cortex. Eur J Neurosci 22:2596-2604.

Fontanini A, Katz DB (2006) State-dependent modulation of time-varying gustatory responses. J Neurophysiol 96:3183-3193.

Frank ME, Formaker BK, Hettinger TP (2003) Taste responses to mixtures: analytic processing of quality. Behav Neurosci 117:228-235.

Gallo M, Roldan G, Bures J (1992) Differential involvement of gustatory insular cortex and amygdala in the acquisition and retrieval of conditioned taste aversion in rats. Behav Brain Res 52:91-97.

Grill HJ, Norgren R (1978) The taste reactivity test. I. Mimetic responses to gustatory stimuli in neurologically normal rats. Brain Res 143:263-279.

Gutierrez R, Carmena JM, Nicolelis MA, Simon SA (2006) Orbitofrontal ensemble activity monitors licking and distinguishes among natural rewards. J Neurophysiol 95:119-133.

Halpern BP, Tapper DN (1971) Taste stimuli: quality coding time. Science 171:1256-1258.

Jones MW, French PJ, Bliss TV, Rosenblum K (1999) Molecular mechanisms of long-term potentiation in the insular cortex in vivo. J Neurosci 19:RC36(1-8).

Katz DB, Steinmetz JE (1997) Single-unit evidence for eye-blink conditioning in cerebellar cortex is altered, but not eliminated, by interpositus nucleus lesions. Learn Mem 4:88-104.

Katz DB, Simon SA, Nicolelis MA (2001a) Dynamic and multimodal responses of gustatory cortical neurons in awake rats. J Neurosci 21:4478-4489.

Katz DB, Simon SA, Nicolelis MAL (2001b) Electrophysiological studies of gustation in awake rats. In: Methods and frontiers in the chemical senses (Simon SA, Nicolelis MAL, eds), pp 339-357. Boca Raton, FL: CRC.

Katz DB, Simon SA, Nicolelis MA (2002) Taste-specific neuronal ensembles in the gustatory cortex of awake rats. J Neurosci 22:1850-1857.

Miranda MI, Ferreira G, Ramirez-Lugo L, Bermudez-Rattoni F (2002) Glutamatergic activity in the amygdala signals visceral input during taste memory formation. Proc Natl Acad Sci USA 99:11417-11422.

Nachman M, Ashe JH (1973) Learned taste aversions in rats as a function of dosage, concentration, and route of administration of LiCl. Physiol Behav 10:73-78.

Nachman M, Ashe JH (1974) Effects of basolateral amygdala lesions on neophobia, learned taste aversions, and sodium appetite in rats. J Comp Physiol Psychol 87:622-643.

Nicolelis MAL, Ghazanfar AA, Faggin BM, Votaw S, Oliveira LMO (1997) Reconstructing the engram: simultaneous, multisite, many single neuron recordings. Neuron 18:529-537.

Nishijo H, Uwano T, Tamura R, Ono T (1998) Gustatory and multimodal neuronal responses in the amygdala during licking and discrimination of sensory stimuli in awake rats. J Neurophysiol 79:21-36.

Nitschke JB, Sarinopoulos I, Mackiewicz KL, Schaefer HS, Davidson RJ (2006) Functional neuroanatomy of aversion and its anticipation. NeuroImage 29:106-116.

Norgren R, Leonard CM (1973) Ascending central gustatory pathways. J Comp Neurol 150:217-237.

Perkel DH, Gerstein GL, Moore GP (1967) Neuronal spike trains and stochastic point processes. II. Simultaneous spike trains. Biophys J 7:419-440.

Quirk GJ, Repa C, LeDoux JE (1995) Fear conditioning enhances short- latency auditory responses of lateral amygdala neurons: parallel recordings in the freely behaving rat. Neuron 15:1029-1039.

Quirk GJ, Armony JL, LeDoux JE (1997) Fear conditioning enhances different temporal components of tone-evoked spike trains in auditory cortex and lateral amygdala. Neuron 19:613-624.

Reilly S, Bornovalova MA (2005) Conditioned taste aversion and amygdala lesions in the rat: a critical review. Neurosci Biobehav Rev 29:1067-1088

Repa JC, Muller J, Apergis J, Desrochers TM, Zhou Y, LeDoux JE (2001) Two different lateral amygdala cell populations contribute to the initiation and storage of memory. Nat Neurosci 4:724-731.

Rollins BL, Stines SG, McGuire HB, King BM (2001) Effects of amygdala lesions on body weight, conditioned taste aversion, and neophobia. Physiol Behav 72:735-742.

Schafe GE, Bernstein IL (1998) Forebrain contribution to the induction of a brainstem correlate of conditioned taste aversion. II. Insular (gustatory) cortex. Brain Res 800:40-47.

Schafe GE, Thiele TE, Bernstein IL (1998) Conditioning method dramatically alters the role of amygdala in taste aversion learning. Learn Mem 5:481-492.

Small DM, Gregory MD, Mak YE, Gitelman D, Mesulam MM, Parrish T (2003) Dissociation of neural representation of intensity and affective valuation in human gustation. Neuron 39:701-711.

Spector AC, Breslin P, Grill HJ (1988) Taste reactivity as a dependent measure of the rapid formation of conditioned taste aversion: a tool for the neural analysis of taste-visceral associations. Behav Neurosci 102:942-952.

Stapleton JR, Lavine ML, Wolpert RL, Nicolelis MA, Simon SA (2006) Rapid taste responses in the gustatory cortex during licking. J Neurosci 26:4126-4138.

Stone ME, Grimes BS, Katz DB (2005) Hippocampal inactivation enhances taste learning. Learn Mem 12:579-586.

Stopfer M, Jayaraman V, Laurent G (2003) Intensity versus identity coding in an olfactory system. Neuron 39:991-1004.

Travers JB, Norgren R (1986) Electromyographic analysis of the ingestion and rejection of sapid stimuli in the rat. Behav Neurosci 100:544-555.

Wang Y-Y, Fontanini A, Katz DB (2006) Temporary basolateral amygdala lesions disrupt acquisition of socially transmitted food preferences in rats. Learn Mem 13:794-800.

Yamamoto T, Azuma S, Kawamura Y (1984) Functional relations between the cortical gustatory area and the amygdala: electrophysiological and behavioral studies in rats. Exp Brain Res 56:23-31.

Yamamoto T, Matsuo R, Kiyomitsu Y, Kitamura R (1988) Sensory inputs from the oral region to the cerebral cortex in behaving rats: an analysis of unit responses in cortical somatosensory and taste areas during ingestive behavior. J Neurophysiol 60:1303-1321.

Yasoshima Y, Yamamoto T (1998) Short-term and long-term excitability changes of the insular cortical neurons after the acquisition of taste aversion learning in behaving rats. Neuroscience 84:1-5.

Yasoshima Y, Shimura T, Yamamoto T (1995a) Single unit responses of the amygdala after conditioned taste aversion in conscious rats. NeuroReport 6:2424-2428

Yasoshima Y, Shimura T, Yamamoto T (1995b) Changes of taste responses in the insular cortex in freely behaving rats after acquisition of conditioned taste aversion learning. Jpn J Physiol 45:S188. 